\title{
Tissue Accumulation of Neutrophil Extracellular Traps Mediates Muscle Hyperalgesia in a Mouse Model
}

\section{Kazuaki Suzuki}

Department of Orthopaedic Surgery, Tohoku University Graduate School of Medicine, Japan Masahiro Tsuchiya ( $\nabla$ tsuchiya-thk@umin.ac.jp )

Tohoku Fukushi University

\section{Shinichiro Yoshida}

Department of Orthopaedic Surgery, Tohoku University Graduate School of Medicine, Japan

\section{Kazumi Ogawa}

Tohoku University Graduate School of Biomedical Engineering

\section{Weijian Chen}

Tohoku University Graduate School of Biomedical Engineering

\section{Makoto Kanzaki}

Tohoku University Graduate School of Biomedical Engineering

\section{Tadahisa Takahashi}

Department of Orthopaedic Surgery, Tohoku University Graduate School of Medicine, Japan

\section{Ryo Fujita}

Department of Orthopaedic Surgery, Tohoku University Graduate School of Medicine, Japan

\section{Yuqing Li}

Tohoku University Graduate School of Biomedical Engineering

\section{Yutaka Yabe}

Department of Orthopaedic Surgery, Tohoku University Graduate School of Medicine, Japan

\section{Toshimi Aizawa}

Department of Orthopaedic Surgery, Tohoku University Graduate School of Medicine, Japan

\section{Yoshihiro Hagiwara}

Department of Orthopaedic Surgery, Tohoku University Graduate School of Medicine, Japan

\section{Research Article}

Keywords: neutrophil extracellular trap, uric acid, muscle pain, citrullinated histone $\mathrm{H} 3$, multiphoton microscopy imaging 
DOI: https://doi.org/10.21203/rs.3.rs-576628/v2

License: (c) (1) This work is licensed under a Creative Commons Attribution 4.0 International License. Read Full License

Version of Record: A version of this preprint was published at Scientific Reports on March 9th, 2022. See the published version at https://doi.org/10.1038/s41598-022-07916-8. 
1 Tissue accumulation of neutrophil extracellular traps mediates muscle

\section{2 hyperalgesia in a mouse model}

4 Kazuaki Suzuki ${ }^{1,2}$, Masahiro Tsuchiya ${ }^{3 *}$, Shinichiro Yoshida ${ }^{1}, K_{a z u m i}$ Ogawa ${ }^{1,2}$, Weijian

5 Chen $^{2}$, Makoto Kanzaki ${ }^{2}$, Tadahisa Takahashi ${ }^{1,2}$, Ryo Fujita ${ }^{1,2}$, Yuqing Li $^{1,2}$, Yutaka Yabe ${ }^{1}$,

6 Toshimi Aizawa ${ }^{1}$, and Yoshihiro Hagiwara ${ }^{1}$

7

$8{ }^{1}$ Department of Orthopaedic Surgery, Graduate School of Medicine, Tohoku University,

9 Sendai, Japan

$10{ }^{2}$ Graduate School of Biomedical Engineering, Tohoku University, Sendai, Japan

$11{ }^{3}$ Department of Nursing, Tohoku Fukushi University, Sendai, Miyagi, Japan

12

$13{ }^{*}$ Corresponding author

14 Masahiro Tsuchiya, DDS., PhD.

15 Department of Nursing, Tohoku Fukushi University

16 6-149-1 Kunimi-ga-oka, Sendai 981-3201, Japan

17 Tel: +81-22-728-6049, Fax: +81-22-233-3113

18 E-mail: tsuchiya-thk@umin.ac.jp

19 


\section{Abstract}

22 Accumulation of uric acid (UA) during muscular trauma is a causative factor involved in

23 the development of muscle hyperalgesia. Neutrophil extracellular traps (NETs), DNA-

24 based reticular structures to capture UA, play a central role in the pain onset of gout

25 attacks; however, the involvement of NETs via the elevation of local UA level in muscle

26 hyperalgesia due to overuse injuries remains unknown. The triceps surae muscles (TSMs)

27 in the unilateral hindlimb of mice were electrically stimulated to induce excessive muscle

28 contraction. Mechanical withdrawal thresholds, tissue UA levels, neutrophil recruitment,

29 protein amount of citrullinated histone 3 (citH3), a major marker of NETs, were

30 investigated. Furthermore, whether neutrophil depletion, extracellular DNA cleavage, and

31 administration of the urate-lowering agent febuxostat could improve muscle hyperalgesia

32 due to NET formation was examined. CitH3 expression upon neutrophil recruitment

33 significantly increased in the stimulated TSMs with an increase in tissue UA levels,

34 whereas febuxostat administration improved muscle hyperalgesia with decreases in citH3

35 and tissue UA levels, as observed in neutrophil depletion and extracellular DNA digestion.

36 The underlying mechanism of muscle hyperalgesia associated with locally recruited

37 neutrophils forming NETs due to the increased tissue UA levels potentially plays a

38 significant role in creating a vicious circle of muscle pain. 
39 Keywords: neutrophil extracellular trap, uric acid, muscle pain, citrullinated histone H3,

40 multiphoton microscopy imaging

\section{Introduction}

43 Skeletal muscle is a common source of pain that markedly impairs activities of daily

44 living ${ }^{1}$. It is widely observed as a major sign of various pathologies, such as neck and

45 shoulder pain ${ }^{2}$, nonspecific lower back pain ${ }^{3}$, and myofascial pain syndrome (MPS) ${ }^{4}$.

46 Based on the higher lifetime prevalence of skeletal muscle pain among the labor

47 population ${ }^{5,6}$, muscle overuse is a key pathogenic event in developing muscle pain.

48 Fundamentally, overuse trauma prompts muscle fibers to release extracellular adenosine

49 triphosphate (ATP), which directly activates pain signaling through purinergic and

50 metabotropic receptors via autocrine and paracrine functions ${ }^{7} 8910$. Indeed, both the

51 serum and muscle tissue levels of uric acid (UA), an end product of purine nucleotides

52 including ATP and dead cell DNA, are reportedly increased due to the production of

53 damaged muscle fibers ${ }^{11,12}{ }^{13-15}$.

54 UA has recently been recognized as a damage-associated molecular pattern (DAMP),

55 which activates an intracellular complex called the inflammasome for processing and

56 releasing interleukin (IL)-1 $\beta$ and IL- $18{ }^{16}$. Our recent study using a muscle pain model by 
57 the repeated electrical stimulation of the triceps surae muscles (TSMs) revealed the

58 marked recruitment of inflammatory cells, including neutrophils and macrophages,

59 producing proinflammatory cytokines, such as IL-1 $\beta$ and IL-18; this was due to

60 inflammasome activation, which was triggered by the increase in tissue UA levels ${ }^{11} 1517$.

61 Furthermore, based on the improvement of muscle hyperalgesia by the administration of

62 xanthine oxidase inhibitors, we suggested that a higher tissue UA concentration could be

63 a causative factor of mechanical hyperalgesia ${ }^{11}$. Thus, the dysregulated innate immune

64 response with hyperuricemia has been associated with gout among various

65 autoinflammatory diseases; these autoinflammatory diseases are characterized by

66 unprovoked episodes of recurrent or continuous inflammation in the absence of high-titer

67 autoantibodies or antigen-specific lymphocytes 181920.

68 A recent study has also supported that the dysregulated innate immune response of

69 neutrophils against monosodium urate (MSU) crystals in the gout flare is a part of the

70 autoinflammatory response because of the involvement of neutrophil extracellular traps

71 (NETs) ${ }^{21}$. The release of NETs, a unique defense mechanism continuing from cell death

72 (NETosis), is regarded as a valuable target for disease pathogenesis in gout ${ }^{21} 2223$. NETs

73 are primarily composed of their own DNA released as reticular structures with an

74 oxidative burst in order to capture and eliminate pathogens, including DAMPs ${ }^{22}$, and 
75 citrullinated histone H3 (citH3) plays a central role in NETosis. Interestingly, NETs also

76 contribute to aggregating neutrophilic proinflammatory mediators, thereby limiting, but

77 prolonging the inflammatory status ${ }^{22}$. Indeed, NETs are not only a DAMP after

78 degradation, but also a potential source of UA with extracellular nucleotide metabolism

$79{ }^{23}$. Thus, research findings regarding the underlying mechanism of UA accumulation with

80 a focus on NETs in damaged muscle tissues will facilitate the development of an

81 integrative therapeutic strategy for chronic muscle pain.

82 Although the involvement of NET-mediated processes in numerous painful diseases,

83 including gout and rheumatoid arthritis, has been reported ${ }^{21} 22$, no reports have described

84 the relationship between NETs and muscle pain. Therefore, using a muscle pain model

85 with muscle overuse, we examined whether neutrophils play a key role in developing

86 muscle pain via NET production triggered by increased UA levels.

87

$88 \quad$ Results

89 MSU stimulation induces NETs and muscle pain in the TSMs

90 To investigate whether MSU crystals induced NETs in skeletal muscle tissues, as shown

91 in previous studies, we first performed the intramuscular injection of MSU by following

92 a method reported in our previous study (Fig. 1) ${ }^{11}$. Compared to those of the control 
93 group, the MNTs of the MSU group significantly decreased after the intramuscular

94 injection of MSU (Fig. 1a). In terms of IHC observations, control TSMs with saline

95 injection did not show any changes, whereas the increased immunoreactivity of citH3 and

96 Gr-1 was located around the outer edge of MSU (Fig. 1b). Western blotting using the

97 TSM tissues stimulated with MSU on day 2 showed significant increases in citH3 (Fig.

98 1c) and Ly6G (Fig. 1d). Additionally, IL-1 $\beta$ expression in the TSMs injected with MSU

99 significantly increased on day 2 (Fig. 1e).

100

101 Excessive muscle contraction caused by repeated EPS induced mechanical

102 hyperalgesia with elevated NET production in the skeletal muscle tissues

103 We next confirmed whether excessive muscle contraction by EPSs induced NET

104 formation associated with an increase in tissue UA levels (Fig. 2). As previously shown

105 in our studies ${ }^{11,17}$, the MNTs of the stimulated TSMs significantly decreased due to

106 repeated stimulation, compared to those of the contralateral muscle, and were

107 significantly decreased, with the minimum MNT noted on day 7 (Fig. 2a). Reduced MNTs

108 in the stimulated TSMs increased again after 7 consecutive days of electrical stimulation,

109 and MNTs between the control (non-stimulated) and MSU (stimulated) groups were not

110 significant on day 11 (Fig. 2a). The tissue samples on day 7 were mainly used in our 
111 analyses based on the above results. Among the TSM samples on day 7, tissue UA levels

112 in the stimulated TSMs were significantly higher than those in the contralateral TSMs

113 (Fig. 2b).

114 IHC analysis indicated distinct distribution patterns of citH3 immunoreactivities,

115 which colocalized with Ly6G immunoreactivities in the stimulated TSMs, but not in the

116 non-stimulated TSMs (Fig. 2c). Hematoxylin and eosin staining showed typical skeletal

117 muscle tissue histology in both groups (data not shown). Further confirming these

118 observations, western blotting indicated that the relative density of citH3 (Fig. 2d) and

119 Ly6G (Fig. 2e) normalized to the loading control (total H3 and GAPDH, respectively)

120 were significantly increased in the stimulated TSMs on day 7 compared with those in the

121 non-stimulated TSMs. The time-series experiment of citH3 protein in comparison with

122 ipsilateral to contralateral TSM tissues during and after EPS is shown in Fig. $2 \mathrm{f}$. The

123 protein amount ratio of citH3 showed the highest value on day 7 with repeated EPS,

124 whereas it recovered to the level on day 0 after stopping the stimulation. IL-1 $\beta$ mRNA

125 expression was significantly increased in the stimulated TSMs than in the non-stimulated

126 TSMs (Fig. 2g).

127

128 DNase treatment ameliorated muscle hyperalgesia and NET induction owing to 
130 To confirm the pathological significance of NETs in muscle hyperalgesia, we

131 administered DNase I to degrade extracellular DNA, including NETs, and demonstrated

132 its impact on muscle hyperalgesia (Fig. 3). Intravenous administration of DNase I

133 significantly increased the MNT values (Fig. 3a) and decreased tissue UA levels (Fig. 3b)

134 in the stimulated TSMs. Consistent with these observations, IHC (Fig. 3c) and western

135 blotting analyses (Fig. 3d and e) indicated that DNase I administration decreased the

136 citH3 expression, but not the protein concentration of Gr-1. Additionally, IL-1 $\beta$ mRNA

137 expression in the stimulated TSMs decreased following DNase I administration (Fig. 3f).

138 Importantly, DNase administration reduced not only citH3, but also tissue UA levels

139 despite the same intensity and duration of the repeated EPS application.

140

141 Neutrophil depletion ameliorated muscle hyperalgesia and NET induction owing to

\section{2 repeated EPS}

143 Given the absolute necessity of neutrophil recruitment for NET induction, we next

144 examined the effects of an experimental neutrophil depletion by prior treatment with anti-

145 Gr-1 antibody on muscle hyperalgesia (Fig. 4). Neutrophil depletion resulted in a

146 significant increase of the MNT in the stimulated hindlimbs (Fig. 4a) and reduced tissue 
147 UA levels (Fig. 4b) in the TSMs with repeated EPS. Similar to the IHC images showing

148 weaker immunoreactivity of citH3 and Gr-1 in the neutrophil-depleted group than in the

149 control group (Fig. 4c), neutrophil depletion significantly decreased the relative ratio of

150 citH3 (Fig. 4d) and IL-1 $\beta$ mRNA expression (Fig. 4e) in the stimulated TSMs compared

151 with the control mice injected with the same amount of normal rat IgG. Notably, the tissue

152 UA level in the stimulated TSMs was decreased with experimental neutrophil depletion.

\section{Febuxostat treatment relieved muscle hyperalgesia due to repeated EPS}

155 Since our recent study reported that the decrease in tissue UA levels resulted in the

156 improvement of muscle hyperalgesia ${ }^{11}$, we investigated whether the administration of

157 febuxostat, a xanthine oxidase inhibitor, influenced NET induction due to repeated EPS

158 (Fig. 5). As reported in our previous study ${ }^{11}$, febuxostat treatment increased the MNTs

159 (Fig. 5a), and reduced tissue UA levels (Fig. 5b) and IL-1 $\beta$ mRNA expression (Fig. 5f) in

160 the stimulated TSMs. Consistent with these observations, IHC (Fig. 5c) and western

161 blotting analyses indicated that febuxostat administration reduced citH3 expression (Fig.

$1625 \mathrm{~d}$ ), but not neutrophil recruitment (Fig. 5c and e) in the stimulated TSMs. These results

163 indicate that high tissue UA levels would play a central role in NET induction in skeletal

164 muscle tissues with hyperalgesia. 


\section{MPM imaging with in vivo staining for NET-like structures}

167 To confirm that repeated EPS induced neutrophil recruitment with extracellular DNA

168 release in TSM tissues, MPM imaging was performed using an in vivo immunostaining

169 method ${ }^{24}$. As shown in Fig. 6 and Supplemental movie, repeated EPS significantly

170 increased the fluorescence of neutrophils and extracellular DNA in TSM tissues, as

171 visualized by QD655 and SYTOX, respectively. In particular, the myofascial region

172 showed significantly more fluorescence colocalizations than the TSM myofibers.

\section{Discussion}

The present study clarified the potential pathogenesis of NETs in muscle hyperalgesia (exhibiting elicited nocifensive behaviors) due to a locally higher tissue UA

177 level caused by excessive muscle contraction (Fig. 2) as well as noted the observations in the TSMs with MSU injection (Fig. 1). Our findings showed a potentially "vicious cycle"

179 model of muscle hyperalgesia due to a higher tissue UA level, which develops in a 180 reciprocal manner between neutrophil recruitment and NET induction (Fig. 7). 
183 late effects on tissues due to the release of cytotoxic and proinflammatory mediators with

184 NET degradation ${ }^{22}{ }^{23}$. Delayed-onset muscle soreness, a typical muscle pain experienced

185 after excessive exercise, persists for a few days ${ }^{7}$. Based on the increased serum NET

186 levels after acute severe exercise ${ }^{25}$, the characteristics of NETs would not only play a

187 crucial role in prolonging and strengthening local inflammation and pain, as observed in

188 our results, but also in general muscle physiology. Additionally, recent studies have

189 focused on the implications of NETs in numerous autoinflammatory diseases, including

190 systemic lupus erythematosus, psoriasis, and gout $2122{ }^{26}$. Interestingly, our results also

191 demonstrated that febuxostat administration, as well as the experimental neutrophil

192 depletion and DNase administration, improved the local NET accumulation associated

193 with muscle hyperalgesia (Fig. 3). Febuxostat was reported to reduce not only tissue UA

194 content, but also neutrophil recruitment in damaged tissues ${ }^{27}$. Thus, local NET

195 accumulation in skeletal muscles damaged by repeated excessive exercise would enhance

196 inflammation and pain with a sustainable supply of proinflammatory stimuli consisting

197 of inflammatory mediators, such as IL-1 $\beta$ and DAMPs, including UA.

198 MSU crystallization due to hyperuricemia is a typical causative stimulus of acute

199 and painful inflammatory responses in gout attacks ${ }^{20}{ }^{21}$. Since our previous work showed

200 that a higher UA level in skeletal muscle tissues was a potential trigger for muscle 
201 hyperalgesia ${ }^{11}$, we here indicated the involvement of NETs as a crucial mediator in

202 developing muscle hyperalgesia caused by a higher UA content in skeletal muscle tissues.

203 However, no MSU crystals were found in the skeletal muscle tissues with excessive

204 contraction. In terms of MSU crystallization, Martillo et al. indicated that $405 \mu \mathrm{mol} / \mathrm{L}$ is

205 the solubility limit of the serum UA ${ }^{28}$. Our results showed that the average local UA

206 concentration (approximately $500 \mu \mathrm{mol} / \mathrm{L}$ ) in the stimulated muscles was higher than the

207 abovementioned value. Because Braga et al. indicated that high concentrations of soluble

208 UA directly activate innate immunity ${ }^{16}$, a higher tissue UA level possibly contributes to

209 the modulation of a microenvironment with a higher risk of NET induction as neutrophil

210 recruitment.

211 In terms of a vicious cycle of a higher tissue UA level leading to NET

212 accumulation and consequent development of muscle hyperalgesia (Fig. 7), both

213 neutrophil depletion (Fig. 4) and DNase treatment (Fig. 3) essentially resulted not only in

214 the improvement of muscle hyperalgesia, but also in the reduction of the tissue UA levels

215 in the damaged skeletal muscles, as well as the febuxostat treatment (Fig. 5). Based on

216 the activation of the inflammasome pathway in macrophages by a higher UA level ${ }^{11}{ }^{16}$,

217 the neutrophil-macrophage interactions, as the main innate immune response,

218 definitively contribute to the deterioration of muscle hyperalgesia. Although future 
219 studies with appropriate designs are warranted to clarify the above aspects, these findings

220 suggest that extracellular DNA consisting of NETs with neutrophil recruitment is

221 potentially the main source inducing a higher UA level in skeletal muscle tissues via

222 purine metabolism and plays a role in exacerbating muscle hyperalgesia.

Furthermore, MPM imaging of NET-like structures showed that extracellular

224 DNA fragments were mostly colocalized with neutrophils, but not in the skeletal muscle

225 fibers. Shinoda et al. also reported no histological changes in the stimulated skeletal

226 muscle tissues under the same experimental conditions ${ }^{10}$. Thus, a higher tissue UA

227 content with skeletal muscle overloading may predominantly depend on neutrophil

228 recruitment followed by NETosis. Furthermore, neutrophils releasing extracellular DNA

229 were predominantly recruited more in myofascial tissues than in muscle fibers (Fig. 6).

230 This is consistent with the multiple lines of evidence regarding the pathomechanisms

231 underlying the development of MPS with the characteristic irritable response in

232 myofascial tissues ${ }^{15}$. Future studies are warranted to clarify the mechanisms that

233 dominantly recruit neutrophils in myofascial tissues, thus providing new insights

234 regarding MPS therapy and management. 
237 Our findings of NET dynamics clarified the underlying mechanism of muscle

238 hyperalgesia associated with recruited neutrophils forming NETs, which potentially cause

239 a higher UA content in skeletal muscle tissues. Thus, the regulation of local UA

240 metabolism while focusing on NET induction would be a potential therapeutic target to

241 relieve muscle pain.

243 Methods

\section{Experimental animals}

245 Male BALB/c mice (5-7 weeks old) were used in this study (CLEA Japan, Tokyo, Japan).

246 The study was carried out in compliance with the ARRIVE guidelines. Further, the

247 experimental design, care, and use of the mice were performed according to the guidelines

248 for animal experiments at Tohoku University. Ethical approval for this study was obtained

249 from the Animal Research Committee of Tohoku University (approval number: 2019

250 MdA-070). The mice were kept in standard cages maintained in an air-conditioned room

251 at $23{ }^{\circ} \mathrm{C} \pm 1{ }^{\circ} \mathrm{C}$ with a 12 -h light-dark cycle with ad libitum access to standard food

252 pellets and tap water, in accordance with the National Standards Relating to the Care and

253 Management of Laboratory Animals and Relief of Pain (Notification No. 88 of the

254 Ministry of the Environment, Japan, April 28, 2006). General anesthesia was induced in 
each mouse by the intraperitoneal injection of medetomidine $(0.3 \mathrm{mg} / \mathrm{kg} ; \mathrm{ZENOAQ}$,

256 Fukushima, Japan), midazolam (4.0 mg/kg; SANDZ, Tokyo, Japan), and butorphanol (5.0

$257 \mathrm{mg} / \mathrm{kg}$; Meiji Seika Pharma Co, Tokyo, Japan). Mice were sacrificed by cervical

258 dislocation under inhalation anesthesia with isoflurane (MSD Animal Health, Kenilworth,

259 NJ, USA).

\section{Repeated electrical stimulation of the triceps surae muscles}

262 Electrical pulse stimulation (EPS) was repeatedly applied to induce excessive muscle

263 contraction of the TSMs, as previously described ${ }^{10} 11{ }^{17}$. Two stainless electrodes (single-

264 stranded stainless steel wire, A-M system, Sequim, WA, USA) were transcutaneously

265 inserted into the proximal and distal ends of the TSMs on the dorsal surface of the

266 hindlimbs under anesthesia. EPS using a STG4004 multichannel system (MCS GmbH,

267 Reutlingen, Germany) was performed on the muscle at $10 \mathrm{~Hz}$ with a $10-\mathrm{V}$ amplitude and

268 a $100-\mu$ s pulse width for 30 min everyday (day 0 through day 6 ). The bilateral hindlimbs

269 were immobilized with full ankle dorsiflexion using a scotch tape to stabilize the static

270 muscle tension during EPS. Electrodes were also applied to the contralateral hindlimbs

271 without EPS. Within $24 \mathrm{~h}$ after the last EPS, the TSMs were collected, immediately frozen

272 in liquid nitrogen, and stored at $-80^{\circ} \mathrm{C}$ until assayed. 
274 Assessment of mechanical nociceptive thresholds

275 The mechanical nociceptive threshold (MNT) was defined as the amount of pressure

276 required to evoke pain-related reactions such as vocalization, struggling, and hindlimb

277 withdrawal. The MNT was evaluated using the Randall-Selitto test (MK-201D Pressure

278 Analgesy-Meter, Muromachi Kikai Co., Tokyo, Japan) ${ }^{29}$. The test was performed with a

279 cone-shaped, 2.6-mm-diameter tip attached to a scale with a display. Pressure, gradually

280 increasing at regular intervals $(10 \mathrm{mmHg} / \mathrm{s})$ with setting the cut-off value to be $300 \mathrm{mmHg}$,

281 was applied to the lateral side of the TSMs ${ }^{11}$. Since circadian rhythm affects pain

282 sensitivity, all MNT measurements were performed in the morning. To avoid bias, the

283 MNT assessments were analyzed by an investigator who was blinded to the experimental

284 conditions.

285

286 Local effect of monosodium urate stimulation

287 To confirm the local effects of MSU on NET induction, recrystallized MSU (No. 133-

288 13432; Wako Pure Chemicals Industries, Osaka, Japan) dissolved in saline was

289 administered to the right TSMs (MSU group), as previously described ${ }^{30}{ }^{31}$. Saline was

290 administered to the contralateral TSMs (control group). The solution (100 $\mu \mathrm{L})$ of 
recrystallized MSU $(200 \mu \mathrm{g})$ was injected under the fascia of the lateral head of the TSMs

292 using a 27-gauge needle. MNTs were assessed on days $0,1,2$, and 4 post-injection. The

293 TSMs were obtained, frozen in liquid nitrogen, and stored at $-80{ }^{\circ} \mathrm{C}$ until assayed.

295 Pharmacological experiments related to neutrophil extracellular trap formation

296 We administered deoxyribonuclease (DNase) I (Wako Pure Chemical Industries, Ltd.,

297 Osaka, Japan; $10 \mathrm{mg}$ per kg body weight [BW] per day) through intravenous injection via 298 the tail vein ${ }^{32}$ or febuxostat (F0847, Tokyo Chemical Industry Co., Ltd., Tokyo, Japan; 5

$299 \mathrm{mg}$ per $\mathrm{kg}$ BW per day) through intraperitoneal injection ${ }^{33}$. The control mice were 300 injected with the same volume of the control vehicle (saline) 15 min prior to the 301 experiment. To deplete neutrophils, anti-granulocyte-differentiation antigen 1 (Gr-1; a major

303 neutrophil marker) antibody (RB6-8C5, rat IgG2b) purified from the culture supernatants

304 of a hybridoma (provided by Dr. R. Coffman) was intravenously administered into the

305 mice (Gr-1 group) once every 3 days at a dose of $5 \mathrm{mg} / \mathrm{kg} \mathrm{BW}^{34}{ }^{35}$, because the injection

306 effectively causes neutropenia for at least a few days ${ }^{36,37}$. The control mice were also

307 injected with an equivalent amount of normal rat IgG (Jackson Laboratories, Bar Harbor, 308 ME) (IgG group). 
310 Measurement of tissue uric acid levels

311 The frozen muscles $(30 \mathrm{mg})$ were homogenized in $300 \mu \mathrm{L}$ of lysis buffer $(30 \mathrm{mM}$ Tris,

$312100 \mathrm{mM}$ sodium chloride, $1 \mathrm{mM}$ ethylenediaminetetraacetic acid, $1 \%$ Triton $\mathrm{X}-100,2.5$

$313 \mathrm{mM}$ sodium fluoride, $2 \mathrm{mM}$ sodium polyphosphate, $1 \mathrm{mM}$ sodium orthovanadate, $1 \mathrm{mM}$

314 phenylmethylsulphonyl fluoride, $10 \mu \mathrm{g} / \mathrm{mL}$ aprotinin, $1 \mu \mathrm{g} / \mathrm{mL}$ pepstatin, and $5 \mu \mathrm{g} / \mathrm{mL}$

315 leupeptin) and then the lysate was centrifuged at $12,000 \times \mathrm{g}$ for $15 \mathrm{~min}$ at $4{ }^{\circ} \mathrm{C}$. The

316 supernatants were collected for the assay of tissue UA levels and western blotting. The

317 tissue UA levels were measured using an assay kit (Cayman Chemical Company, Ann

318 Arbor, USA) following the manufacturer's instructions.

320 Quantitative reverse transcription polymerase chain reaction

321 Total RNA was extracted from the TSM tissues using TRIzol (Molecular Research Centre

322 Inc., Cincinnati, OH, USA). cDNA was prepared using a Transcriptor First Strand cDNA

323 Synthesis Kit (Roche, Basel, Switzerland). The primer sequences used were as follows:

324 IL-1b: F5'-TGG TGG GGG TTC TCT GTG GTT-3' and R' '-TTG AGG CGG CTT TCT

325 TTG TCC-3' and EF1a1 (internal control primer): F5'-TCG CTT TGC TGT TCG TGA

326 C-3' and R5'-TGG GGT GGC AGG TGT TAG-3'. The relative expression levels of each 
327 mRNA were calculated as a function of EF1a1 expression, as previously described ${ }^{11} 17$.

329 Immunohistochemistry

330 The TSM tissues were snap-frozen in liquid nitrogen and embedded in a Tissue-Tek

331 OCT compound (Sakura Finetek, Tokyo, Japan). The cryosections were transversely cut

332 into 5- $\mu \mathrm{m}$ thickness using a cryostat (CM1850; Leica, Nussloch, Germany) and

333 mounted on coated glass slides, followed by acetone fixation. After washing and

334 incubating with a blocking buffer (5\% bovine serum albumin [BSA] in Tris-buffered

335 saline with $0.1 \%$ Tween-20) including 10\% normal goat serum (Nichirei Biosciences

336 Inc., Tokyo, Japan) for $30 \mathrm{~min}$. The slides were incubated with a polyclonal rabbit anti-

337 citH3 antibody (ab5103; 10 ng/mL; Abcam, Waltham, MA, USA) and anti-Gr-1

338 antibody (RB6-8C5, rat IgG2b; $10 \mathrm{ng} / \mathrm{mL}$; BioLegend, San Diego, CA, USA) in a

339 blocking buffer for $2 \mathrm{~h}$ at room temperature (RT). Subsequently, the slides were

340 incubated for $1 \mathrm{~h}$ with an Alexa Fluor 488-conjugated goat anti-rabbit IgG (A-11034,

341 Life Technologies, Carlsbad, CA, USA; dilution, 1:750) for anti-citH3 and an Alexa

342 Fluor 555-conjugated goat anti-rat IgG (A- 21434, Life Technologies; dilution, 1:750)

343 for Gr-1 at RT. After washing, the slides were incubated with 4,6-diamidino-2-

344 phenylindole (Sigma-Aldrich; dilution, 1:500) for nuclear staining. Images were 
captured using a fluorescence microscope (Olympus FV1000; Olympus, Tokyo, Japan)

346 equipped with an oil-immersion objective lens (UApo/340 40×/NA 1.35). The images

347 were analyzed using Fiji/ImageJ software (NIH, Bethesda, MD, USA). At least three

348 images from each slide were captured at 200X magnification. To avoid bias, a few

349 animals were used for immunohistochemistry (IHC), and two slides/animals were

350 analyzed. After confirming the reproducibility, representative images were obtained.

352 Immunoblotting

353 The lysates extracted from the TSM tissues were adjusted to $4.0 \mathrm{mg} / \mathrm{mL}$ with a lysis buffer

354 using a BCA Protein Assay Kit (Thermo Fisher Scientific, Waltham, MA, USA). In brief,

$35530 \mu \mathrm{L}$ of samples were loaded for 5\%-12\% SDS-polyacrylamide gel electrophoresis and

356 transferred to Immobilon-P polyvinylidene difluoride membranes (Merck, Kenilworth,

357 NJ, USA) ${ }^{38}$. The protein-transferred membranes were blocked with 5\% BSA in Tris-

358 buffered saline with $0.1 \%$ Tween-20 and then incubated at $4{ }^{\circ} \mathrm{C}$ overnight with anti-citH3

359 (ab5103; $2 \mu \mathrm{g} / \mathrm{mL})$, anti-histone H3 (ab1791; $1 \mu \mathrm{g} / \mathrm{mL} ;$ Abcam), anti-Ly6G (127602; 2

$360 \mu \mathrm{g} / \mathrm{mL}$; Biolegend), and anti-GAPDH (\#2118; 1:1000 dilution; Cell Signaling, Beverly,

361 MA, USA). The membranes were washed, followed by incubation at RT for $1 \mathrm{~h}$ with

362 HRP-conjugated secondary antibodies (1:10,000 dilutions of \#ab6734; Abcam; and 
363 1:5,000 dilutions of \#32460; Thermo Fisher Scientific). Next, a signal using a

364 chemiluminescence reagent, was obtained from SuperSignal West Femto Maximum

365 Sensitivity Substrate (Thermo Fisher Scientific), and the band intensity was detected

366 using the Image Quant TL system (GE Healthcare, Chalfont St Giles, UK).

\section{Multiphoton microscopy imaging using in vivo staining of NET-like structures}

369 To detect NETs through in vivo staining, two types of fluorescent reagents, anti-Gr-1

370 antibody conjugated to Qdot655 (QD-Gr-1Ab, $3.0 \mu \mathrm{g}$ per mouse) to detect neutrophils

371 and SYTOX Green (Thermo Fisher Scientific, $1 \mu \mathrm{L}$ of $5 \mathrm{mM}$ solution per mouse) to detect

372 extracellular DNA, were used $383^{24}$, and anti-Gr-1 antibodies were prepared by

373 conjugation to Qdot 655 using the SAIVI rapid antibody labeling kit (Invitrogen). The

374 conjugated antibodies were purified using a size-exclusion column, and its concentration

375 was determined by measuring the absorbance at $679 \mathrm{~nm}$. After $24 \mathrm{~h}$ from EPS on day 7,

376 QD-Gr-1Ab and SYTOX were intravenously injected at 20 min before animal sacrifice

377 under anesthesia. Mice were fixed by transcardiac perfusion with $4 \%$ paraformaldehyde

378 in PBS, and the TSM tissues were subjected to multiphoton microscopy (MPM) imaging.

379 An upright A1R-MP multiphoton microscope (Nikon) equipped with a Ti-sapphire laser

380 (Mai-Tai Deep See, Spectra-Physics), GaAsP non-descanned detectors, and a water- 
381 immersion objective lens (CFP75 Apo LWD 25x/NA1.1) was used for image recording

382 with an excitation laser consistently set at $920 \mathrm{~nm}$, with an area size of $510 \mu \mathrm{m} \times 510 \mu \mathrm{m}$

383 and a resolution of $600 \mathrm{dpi}$. The wavelengths for detection using emission filter cubes

384 were 492/SP nm for second-harmonic generation signals (blue channel), $525 \pm 50 \mathrm{~nm}$ for

385 SYTOX (green channel), and $629 \pm 56 \mathrm{~nm}$ for QD-Gr-1Ab (far-red channel). By

386 distinguishing between the muscle and myofascial area based on the second-harmonic

387 generation signals indicating muscle and collagenous fibers ${ }^{40}$, we obtained the

388 fluorescence colocalization of QD-Gr-1Ab and SYTOX to quantitatively measure the

389 accumulation of NET-like structures in each area using ImageJ software ${ }^{41}$. Further,

390 representative 3D image stacks focusing on neutrophil recruitment releasing extracellular

391 DNA were reconstructed from the sequential images in TSM structure.

\section{Statistical analysis}

394 Statistical analyses were performed using SPSS (IBM, Armonk, NY, USA). Analysis of

395 the MNT time-course data was performed using two-way analysis of variance (ANOVA),

396 and repeated measurements were compared using Tukey's post-hoc multiple-comparison

397 test. To compare data from more than two groups from single and multiple days, one-way

398 and two-way ANOVA with Tukey's post-hoc multiple comparison test were used for the 

paired t-tests. All data are expressed as the mean \pm standard error. Statistical significance

\section{References}

4051 Fayaz, A., Croft, P., Langford, R. M., Donaldson, L. J. \& Jones, G. T. Prevalence 406 of chronic pain in the UK: a systematic review and meta-analysis of population 407 studies. BMJ open 6, e010364, doi:10.1136/bmjopen-2015-010364 (2016).

4082 Hanvold, T. N. et al. The effect of work-related sustained trapezius muscle activity 409 on the development of neck and shoulder pain among young adults. Scandinavian 410 journal of work, environment \& health 39, 390-400, doi:10.5271/sjweh.3357 411 (2013).

4123 van Dieen, J. H., Selen, L. P. \& Cholewicki, J. Trunk muscle activation in low413 back pain patients, an analysis of the literature. Journal of electromyography and $414 \quad$ kinesiology : official journal of the International Society of Electrophysiological $415 \quad$ Kinesiology 13, 333-351, doi:10.1016/s1050-6411(03)00041-5 (2003).

4164 Borg-Stein, J. \& Simons, D. G. Focused review: myofascial pain. Archives of 417 physical medicine and rehabilitation $\mathbf{8 3}, \quad \mathrm{S} 40-47, \quad \mathrm{~S} 48-49$, 418 doi:10.1053/apmr.2002.32155 (2002).

4195 Punnett, L. \& Wegman, D. H. Work-related musculoskeletal disorders: the 420 epidemiologic evidence and the debate. Journal of electromyography and 421 kinesiology : official journal of the International Society of Electrophysiological

4236 Mills, S. E. E., Nicolson, K. P. \& Smith, B. H. Chronic pain: a review of its 424 epidemiology and associated factors in population-based studies. British journal 425 4267 Mense, S. Muscle pain: mechanisms and clinical significance. Deutsches 427 Arzteblatt international 105, 214-219, doi:10.3238/artzebl.2008.0214 (2008).

4288 Mastaglia, F. L. The relationship between muscle pain and fatigue. 

doi:10.1016/j.nmd.2012.10.003 (2012).

4319 Dubin, A. E. \& Patapoutian, A. Nociceptors: the sensors of the pain pathway. The Journal of clinical investigation 120, 3760-3772, doi:10.1172/JCI42843 (2010).

43310 Shinoda, M., Ozaki, N. \& Sugiura, Y. Involvement of ATP and its receptors on 434 nociception in rat model of masseter muscle pain. Pain 134, 148-157, 436 doi:10.1016/j.pain.2007.04.006 (2008).

437

11 Yoshida, S. et al. Involvement of inflammasome activation via elevation of uric acid level in nociception in a mouse model of muscle pain. Molecular pain 15, 1744806919858797, doi:10.1177/1744806919858797 (2019).

440

441

442

13 Green, H. J. \& Fraser, I. G. Differential effects of exercise intensity on serum uric 443

12 Retamoso, L. T. et al. Increased xanthine oxidase-related ROS production and TRPV1 synthesis preceding DOMS post-eccentric exercise in rats. Life sciences 152, 52-59, doi:10.1016/j.lfs.2016.03.029 (2016).

446

447

448 acid concentration. Med Sci Sports Exerc 20, 55-59, doi:10.1249/00005768198802000-00008 (1988).

14 Kono, H., Chen, C. J., Ontiveros, F. \& Rock, K. L. Uric acid promotes an acute inflammatory response to sterile cell death in mice. The Journal of clinical investigation 120, 1939-1949, doi:10.1172/JCI40124 (2010).

15 Chen, R., Yin, C., Fang, J. \& Liu, B. The NLRP3 inflammasome: an emerging therapeutic target for chronic pain. Journal of neuroinflammation 18, 84, doi:10.1186/s12974-021-02131-0 (2021).

452

453

17 Yoshida, S. et al. Involvement of neutrophils and interleukin-18 in nociception in 454 a mouse model of muscle pain. Molecular pain

14, 1744806918757286, doi:10.1177/1744806918757286 (2018). in Disease: Potential Anti-NETs Therapeutics. Clinical reviews in allergy \& immunology, doi:10.1007/s12016-020-08804-7 (2020).

20 Punzi, L., Scanu, A., Ramonda, R. \& Oliviero, F. Gout as autoinflammatory disease: new mechanisms for more appropriated treatment targets. Autoimmunity reviews 12, 66-71, doi:10.1016/j.autrev.2012.07.024 (2012). 
46521 Bodofsky, S., Merriman, T. R., Thomas, T. J. \& Schlesinger, N. Advances in our 466 understanding of gout as an auto-inflammatory disease. Seminars in arthritis and

467

468

469

470

471

472

473

474

475

476

477

478

479

480

481

482

483

484

485

486

487

488

489

490

491

492

493

494

495

4963

497

498

499

500 rheumatism 50, 1089-1100, doi:10.1016/j.semarthrit.2020.06.015 (2020).

22 Neubert, E. et al. Chromatin swelling drives neutrophil extracellular trap release. Nature communications 9, 3767, doi:10.1038/s41467-018-06263-5 (2018).

23 Cicco, S., Cicco, G., Racanelli, V. \& Vacca, A. Neutrophil Extracellular Traps (NETs) and Damage-Associated Molecular Patterns (DAMPs): Two Potential Targets for COVID-19 Treatment. Mediators of inflammation 2020, 7527953, doi:10.1155/2020/7527953 (2020).

24 Sumagin, R., Prizant, H., Lomakina, E., Waugh, R. E. \& Sarelius, I. H. LFA-1 and Mac-1 define characteristically different intralumenal crawling and emigration patterns for monocytes and neutrophils in situ. Journal of immunology (Baltimore, Md. : 1950) 185, 7057-7066, doi:10.4049/jimmunol.1001638 (2010).

25 Syu, G. D., Chen, H. I. \& Jen, C. J. Acute severe exercise facilitates neutrophil extracellular trap formation in sedentary but not active subjects. Med Sci Sports Exerc 45, 238-244, doi:10.1249/MSS.0b013e31826df4a1 (2013).

26 Gray, R. D. et al. Delayed neutrophil apoptosis enhances NET formation in cystic fibrosis. Thorax 73, 134-144, doi:10.1136/thoraxjnl-2017-210134 (2018).

27 Kataoka, H., Yang, K. \& Rock, K. L. The xanthine oxidase inhibitor Febuxostat reduces tissue uric acid content and inhibits injury-induced inflammation in the liver and lung. European journal of pharmacology 746, 174-179, doi:10.1016/j.ejphar.2014.11.013 (2015).

28 Martillo, M. A., Nazzal, L. \& Crittenden, D. B. The crystallization of monosodium urate. Current rheumatology reports 16, 400, doi:10.1007/s11926-013-0400-9 (2014).

29 Randall, L. O. \& Selitto, J. J. A method for measurement of analgesic activity on inflamed tissue. Archives internationales de pharmacodynamie et de therapie 111, 409-419 (1957).

30 Ju, T.-J., Dan, J.-M., Cho, Y.-J. \& Park, S.-Y. Inhibition of Inducible Nitric Oxide Synthase Attenuates Monosodium Urate-induced Inflammation in Mice. kjpp 15, 363-369, doi:10.4196/kjpp.2011.15.6.363 (2011).

31 Chen, C.-J. et al. MyD88-dependent IL-1 receptor signaling is essential for gouty inflammation stimulated by monosodium urate crystals. The Journal of clinical investigation 116, 2262-2271, doi:10.1172/JCI28075 (2006).

32 Cagliani, J., Yang, W. L., Brenner, M. \& Wang, P. Deoxyribonuclease Reduces Tissue Injury and Improves Survival After Hemorrhagic Shock. The Journal of 
surgical research 249, 104-113, doi:10.1016/j.jss.2019.11.036 (2020).

50233 Wang, S. et al. Febuxostat pretreatment attenuates myocardial 503 ischemia/reperfusion injury via mitochondrial apoptosis. Journal of translational $504 \quad$ medicine 13, 209, doi:10.1186/s12967-015-0578-x (2015).

50534 Chen, L., Watanabe, T., Watanabe, H. \& Sendo, F. Neutrophil depletion 506 exacerbates experimental Chagas' disease in BALB/c, but protects C57BL/6 mice through modulating the Th1/Th2 dichotomy in different directions. European journal of immunology 31, 265-275, doi:10.1002/15214141(200101)31:1<265::Aid-immu265>3.0.Co;2-1 (2001).

$51035 \quad$ Hirano, Y. et al. Neutralization of osteopontin attenuates neutrophil migration in sepsis-induced acute lung injury. Critical care (London, England) 19, 53,

51336 Conlan, J. W. \& North, R. J. Neutrophils are essential for early anti-Listeria defense in the liver, but not in the spleen or peritoneal cavity, as revealed by a granulocyte-depleting monoclonal antibody. The Journal of experimental medicine 179, 259-268, doi:10.1084/jem.179.1.259 (1994).

37 Rakhmilevich, A. L. Neutrophils are essential for resolution of primary and secondary infection with Listeria monocytogenes. Journal of leukocyte biology 57, 827-831, doi:10.1002/jlb.57.6.827 (1995).

38 Chaweewannakorn, C. et al. Exercise-evoked intramuscular neutrophilendothelial interactions support muscle performance and GLUT4 translocation: a mouse gnawing model study. The Journal of physiology 598, 101-122, doi:10.1113/JP278564 (2020).

39 Masuda, S. et al. Measurement of NET formation in vitro and in vivo by flow cytometry. Cytometry. Part A : the journal of the International Society for Analytical Cytology 91, 822-829, doi:10.1002/cyto.a.23169 (2017).

40 Chaweewannakorn, C. et al. Imaging of muscle activity-induced morphometric changes in fibril network of myofascia by two-photon microscopy. Journal of anatomy 238, 515-526, doi:10.1111/joa.13339 (2021).

41 Tsuchiya, M. et al. Neutrophils Provide a Favorable IL-1-Mediated Immunometabolic Niche that Primes GLUT4 Translocation and Performance in Skeletal Muscles. Cell reports 23, 2354-2364, doi:10.1016/j.celrep.2018.04.067 
536 The authors are grateful to Dr. Shigenori Sekiai, Dr. Chayanit Chaweewannakorn, and

537 Ms. Natsumi Emoto at the Tohoku University Graduate School of Biomedical

538 Engineering for their technical assistance and Dr. Hiroyuki Tada at the Tohoku

539 University Graduate School of Dentistry for the excellent advice on the study. We also

540 thank other doctors at the Department of Orthopaedic Surgery, Tohoku University

541 Graduate School of Medicine, for their excellent technical advice in this study.

543 Authors' contributions

544 Conceptualization: KS, MT, SY, MK, and YH; Data curation: KS, MT, and KO; Formal

545 analysis: KS and MT; Funding acquisition: MT, SY, MK, and YH; Investigation: KS,

546 SY, KO, WC, TT, RF, and YL; Methodology: KS, MT, WC, MK, and YL; Project

547 administration: MT, SY, MK, YY, and YH; Resources: KS, MT, SY, KO, WC, TT, RF,

548 and YL; Supervision: MK, TA, and YH; Validation: TT, RF, and YL; Writing - original

549 draft: KS, MT, SY, and YH. All authors contributed to the data analysis and work

550 presented in this paper.

551

552 Declarations

553 Ethics approval and consent to participate 
554 The experimental design, care, and use of the mice were performed according to the

555 guidelines for animal experiments at Tohoku University. Ethical approval for this study

556 was obtained from the Animal Research Committee of Tohoku University (approval

557 number: 2019 MdA-070).

558 Consent for publication

559 Not applicable

560 Availability of data and materials

561 The data that support the findings of this study are available from the corresponding

562 author, MT, upon reasonable request.

\section{Competing interests}

564 The authors declare that they have no competing interests.

565 Funding

566 This work was supported in part by grants from the Japan Society for the Promotion of

567 Science (nos. 16K11580 and 19K10229 to MT).

568

$569 \quad$ Figure Legends

570 Figure 1. Induction of muscle hyperalgesia and NETs with intramuscular MSU injection.

571 (a) Time-series experiment of MNT values in the MSU- or Saline-injected (contralateral) 
572 hindlimbs. (b) Representative IHC images of TSM tissue on day 2, indicating citH3

573 (green), Gr-1 (red), and DAPI (blue). Western blotting analysis of citH3 (c) and Ly6G (d)

574 amounts in TSM tissues on day 2 after intramuscular injection of MSU or Saline. The

575 relative density is normalized based on the loading control (total histone H3: total H3 and

576 GAPDH, respectively). Scale bar $=100 \mu \mathrm{m}$. (e) qRT-PCR analysis was performed to

577 evaluate expression levels of IL-1 $\beta$, using MSU- or saline-injected TSM tissues on day 2.

578 All data are shown as mean \pm SE $(n=6-10)$ and analysed using paired t-test or two-way

579 ANOVA with Tukey's post-hoc multiple comparison test for time-series of MNT values.

580 Statistical significance is indicated with * $(\mathrm{p}<0.05),{ }^{* *}(\mathrm{p}<0.01)$ and $* * *(\mathrm{p}<0.001)$,

581 respectively.

582

583 Figure 2. Effects of repeated electrical stimulation (EPS) to induce muscle hyperalgesia

584 and NETs. (a) Time series experiment of MNT values in the stimulated and non-

585 stimulated hindlimbs (contralateral side in the same mice). (b) The uric acid level in the

586 stimulated and non-stimulated TSM tissues on day 7. (c) Representative IHC images of

587 mouse TSM tissue with or without repeated EPS on day 7, indicating citH3 (green), Gr-

5881 (red), and DAPI (blue). Scale bar $=100 \mu \mathrm{m}$. (d) citH3 and (e) Ly6G amounts in TSM

589 tissues with or without repeated EPS on day 7 by western blotting analysis. The relative 
590 density is normalized based on the loading control (total histone H3: total H3 and GAPDH,

591 respectively). (f) Time series experiment of citH3 induction in stimulated TSM tissues.

592 After normalization of citH3 using the total $\mathrm{H} 3$, a ratio of citH3 in ipsilateral side relative

593 to contralateral side was calculated in each individual. (g) qRT-PCR analysis was

594 performed to evaluate expression levels of IL-1 $\beta$, using TSM tissues of control or

595 stimulated mice on day 7. All data are shown as mean $\pm \operatorname{SE}(n=4-9)$ and analysed using

596 paired t-test for comparing the mean of two different samples or two-way ANOVA

597 followed by Tukey's post-hoc multiple comparison test for time-series experiment of

598 MNT values and for comparing more than two groups. Statistical significance is indicated

599 with $*(\mathrm{p}<0.05), * *(\mathrm{p}<0.01)$ and $* * *(\mathrm{p}<0.001)$, respectively.

600

601 Figure 3. Effects of DNase treatment on NETs induction associated with muscle

602 hyperalgesia. MNT values of hindlimbs (a) and the uric acid level (b) in TSM tissues in

603 sham-stimulated control and stimulated mice injected intravenously with $10 \mathrm{mg} / \mathrm{kg} / \mathrm{day}$

604 of DNase I or a vehicle. (c) Representative IHC images of the stimulated TSM tissue with

605 or without DNase I administration on day 7, indicating citH3 (green), Gr-1 (red), and

606 DAPI (blue). Scale bar $=100 \mu \mathrm{m}$. Western blotting analysis of citH3 (d) and Ly6G (e)

607 amounts in TSM tissues on day 7. The relative density is normalized based on the loading 
608 control (total H3 and GAPDH, respectively), and further calculated as a ratio in ipsilateral

609 side relative to contralateral (unstimulated) side of the same individual. (f) qRT-PCR

610 analysis was performed to evaluate expression levels of IL-1 $\beta$ in TSM tissues on day 7.

611 All data are shown as mean $\pm \operatorname{SE}(n=4-9)$ and analysed using unpaired t-test for

612 comparing the mean of two different samples or two-way ANOVA followed by post-hoc

613 Tukey's test for a comparison of more than two groups. Statistical significance is

614 indicated with $*(\mathrm{p}<0.05), * *(\mathrm{p}<0.01)$ and $* * *(\mathrm{p}<0.001)$, respectively.

615

616 Figure 4. Effects of neutrophil depletion on NETs induction associated with muscle

617 hyperalgesia. MNT values of hindlimbs (a) and the uric acid level (b) in TSM tissues in

618 sham-stimulated control and stimulated mice injected intravenously with $5 \mathrm{mg} / \mathrm{kg} \mathrm{BW}$ of

619 anti-Gr-1 antibodies or control rat IgGs every 3 days. (c) Representative IHC images of

620 the stimulated TSM tissue with or without neutrophil depletion on day 7, indicating citH3

621 (green), Gr-1 (red), and DAPI (blue). Scale bar $=100 \mu \mathrm{m}$. (d) Western blotting analysis

622 of citH3 amounts in TSM tissues on day 7. The relative density is normalized based on

623 the loading control (total H3), and further calculated as a ratio in ipsilateral side relative

624 to contralateral (unstimulated) side of the same individual. (e) qRT-PCR analysis was

625 performed to evaluate expression levels of IL-1 $\beta$ in TSM tissues on day 7. All data are 
626 shown as mean \pm SE $(n=4-8)$ and analysed using unpaired t-test for comparing the

627 mean of two different samples or two-way ANOVA followed by post-hoc Tukey's test for

628 a comparison of more than two groups. Statistical significance is indicated with * $(\mathrm{p}<$

$6290.05), * *(p<0.01)$ and $* * *(p<0.001)$, respectively.

630

631 Figure 5. Effects of febuxostat treatment on NETs induction associated with muscle

632 hyperalgesia. MNT values of hindlimbs (a) and the uric acid level (b) in TSM tissues in

633 sham-stimulated control and stimulated mice injected intraperitoneally with $5 \mathrm{mg} / \mathrm{kg}$

634 BW/day of febuxostat or a vehicle. (c) Representative IHC images of the stimulated TSM

635 tissue with or without febuxostat treatment on day 7, indicating citH3 (green), Gr-1 (red),

636 and DAPI (blue). Scale bar $=100 \mu \mathrm{m}$. (d) Western blotting analysis of citH3 amounts in

637 TSM tissues with or without febuxostat treatment on day 7. The relative density is

638 normalized based on the loading control (total H3), and further calculated as a ratio in

639 ipsilateral side relative to contralateral (unstimulated) side of the same individual. (e)

640 qRT-PCR analysis was performed to evaluate expression levels of IL-1 $\beta$ in TSM tissues

641 on day 7 . All data are shown as mean $\pm \operatorname{SE}(n=4-9)$ and analysed using unpaired t-test

642 for comparing the mean of two different samples or two-way ANOVA followed by post-

643 hoc Tukey's test for a comparison of more than two groups. Statistical significance is 
644 indicated with $*(\mathrm{p}<0.05), * *(\mathrm{p}<0.01)$ and $* * *(\mathrm{p}<0.001)$, respectively.

645

646 Figure 6. MPM imaging of neutrophil recruitment releasing extracellular DNA due to

647 repeated electrical stimulation (EPS) as assessed by in vivo immunostaining.

648 Representative images show fluorescence colocalization of neutrophils (indicated by

649 white arrows) labelled with QD655-conjugated anti-Gr-1 antibody (red) and extracellular

650 DNA labelled with SYTOX (green) in TSM tissues (muscle fibers and myofascia zone)

651 of sham-control or stimulated mice on day 7. Muscle and collagenous fibers are visualized

652 with second-harmonic generation (blue). The graph summarizing area quantification (\%)

653 of fluorescence colocalizations within myofascia or muscle fibers of TSM tissues is

654 indicated. Data are shown as the mean \pm SE $(n=3)$. The quantification results were

655 analysed using one-way ANOVA with Tukey's post hoc test. Scale bar $=50 \mu \mathrm{m}$. Both a

656 and $\mathrm{b}$ show significant difference to all other groups at $\mathrm{P}<0.05$, respectively.

657

658 Figure 7. A simple schema of the developing pathway of muscle hyperalgesia caused by

659 a higher tissue uric acid level in the mouse model, and the effects of inhibitors at each

660 factor.

661 
662 Figure 1

663

(a)

664

665

666

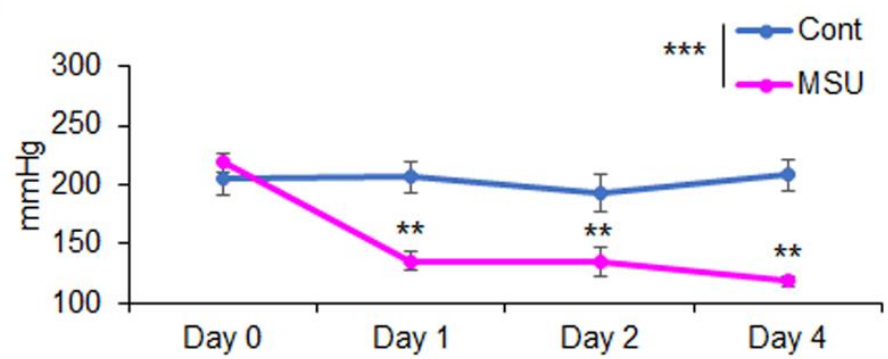

MSU $\uparrow$

(b)
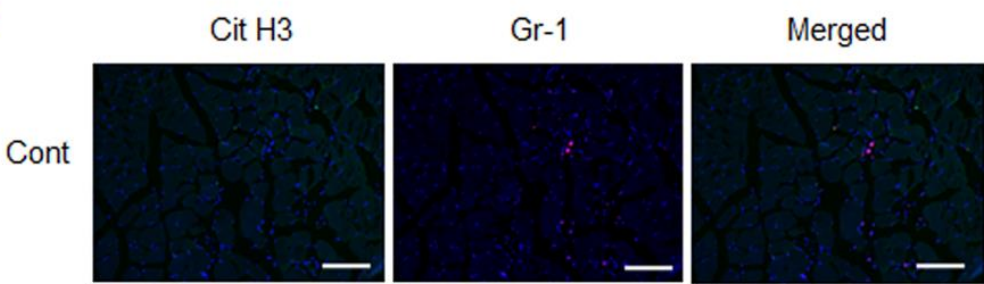

669

670

MSU
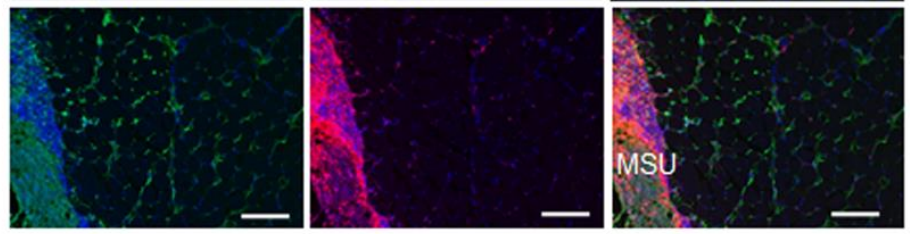

671

(c)

672

673

674

675

676

677 (d)
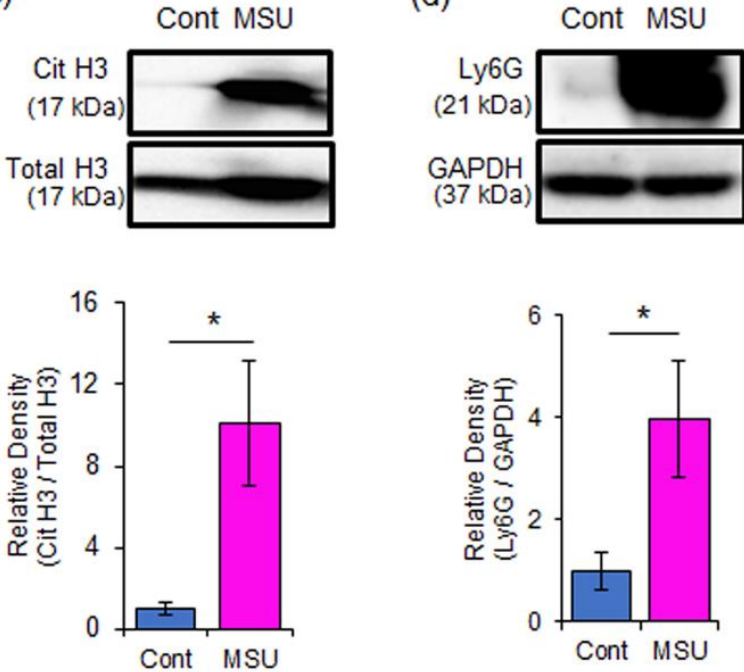

(e)

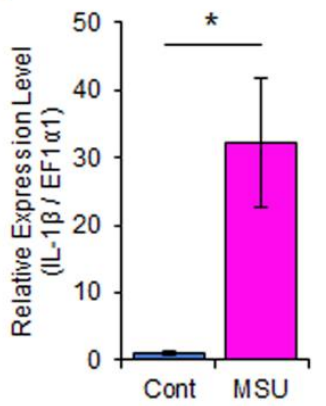

678 
Figure 2

680

681

682

(a)

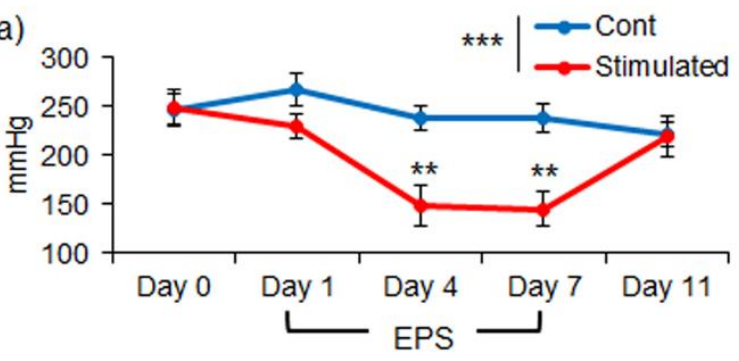

(b)

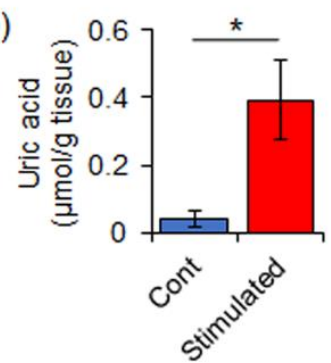

683

(c)

Gr-1

Merged

684
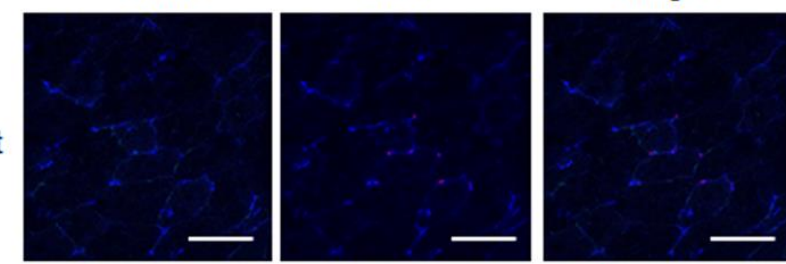

686

Stimulated

687
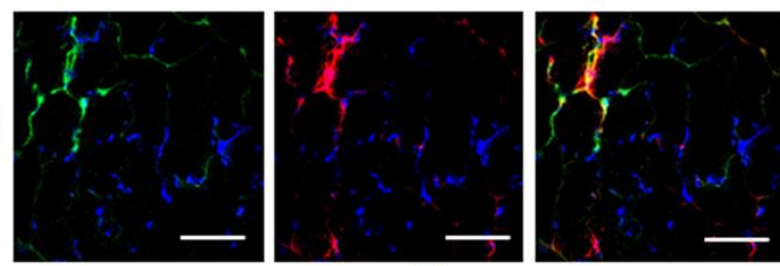

(d)

688

689

690

(f)

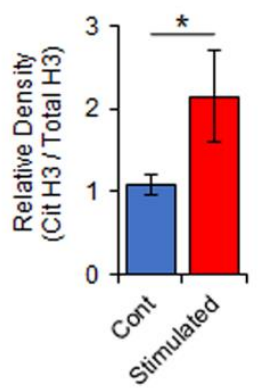

(e)

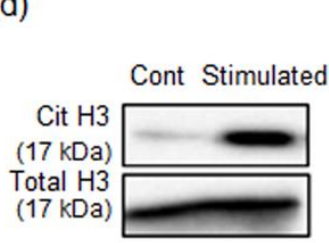

(17 kDa)

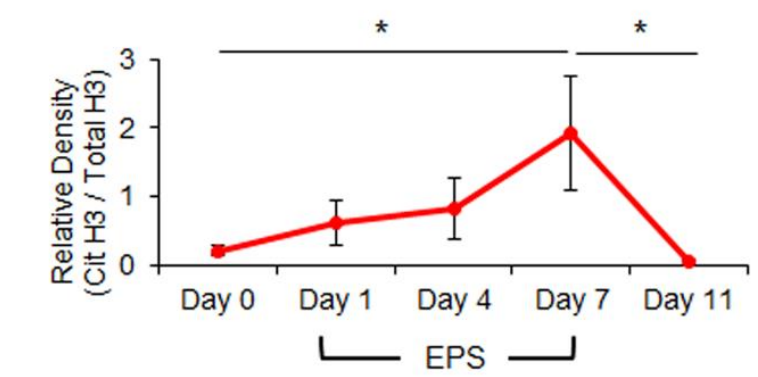

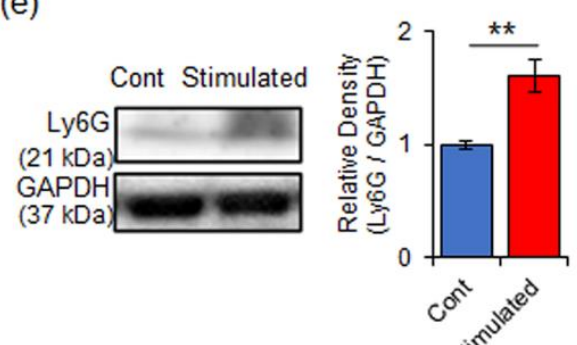

(g)

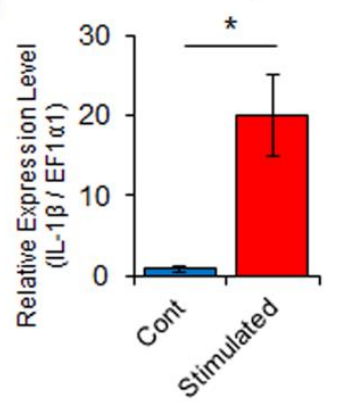

694

695 
$696 \quad$ Figure 3

697 (a)

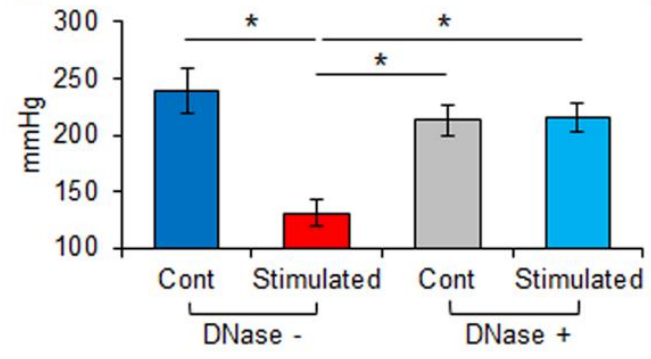

(b)

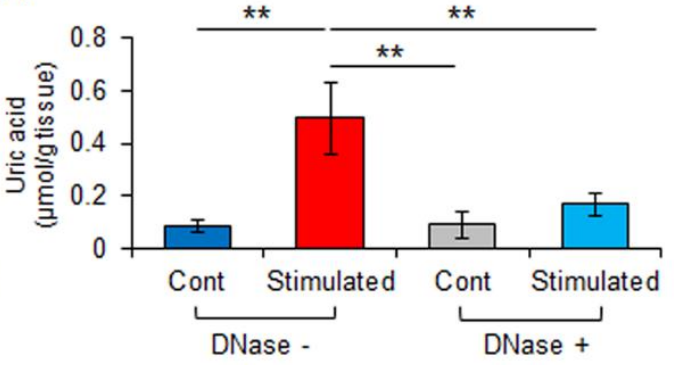

700 (c)

(c)

701

702

DNase -

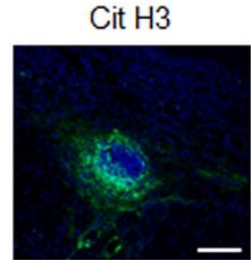

Gr-1

Merged
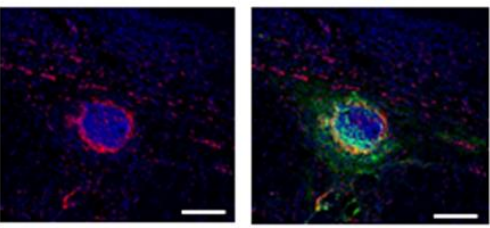

DNase +
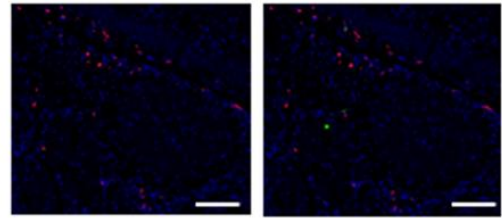

(d)

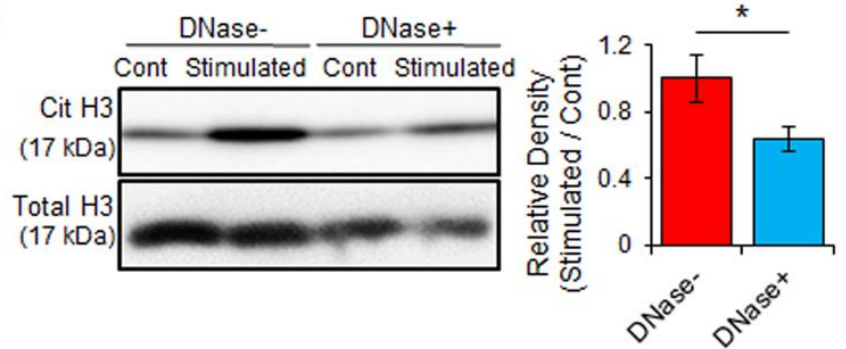

(e)

DNase- DNase+

708

709

710

(f)
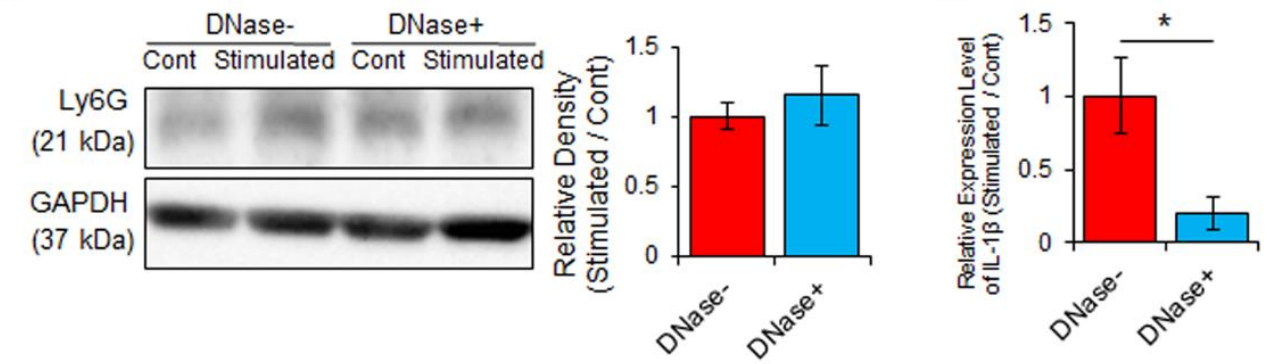

711 
713

714

715

716

717

718

719

720

721

722

723

724

725

726

727
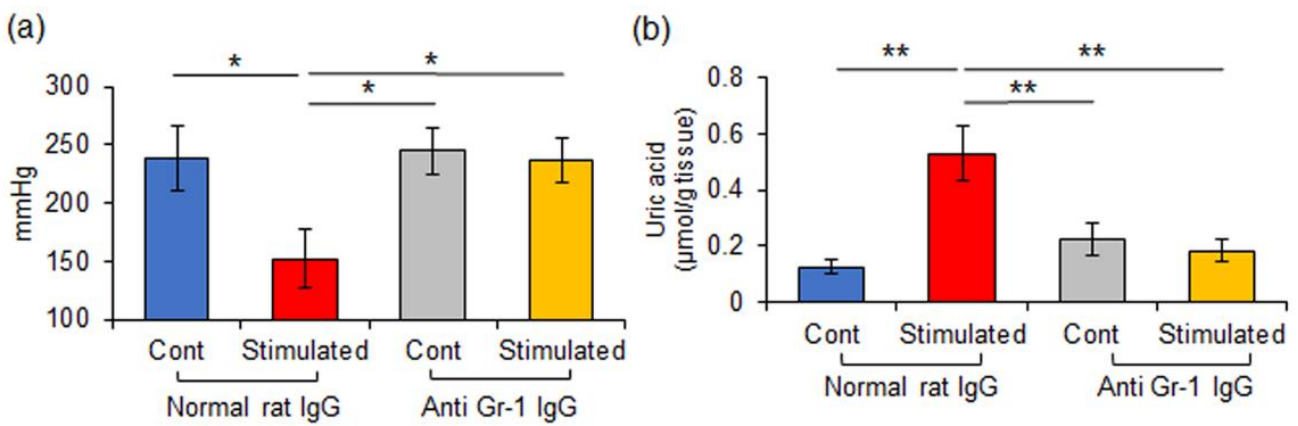

(c)
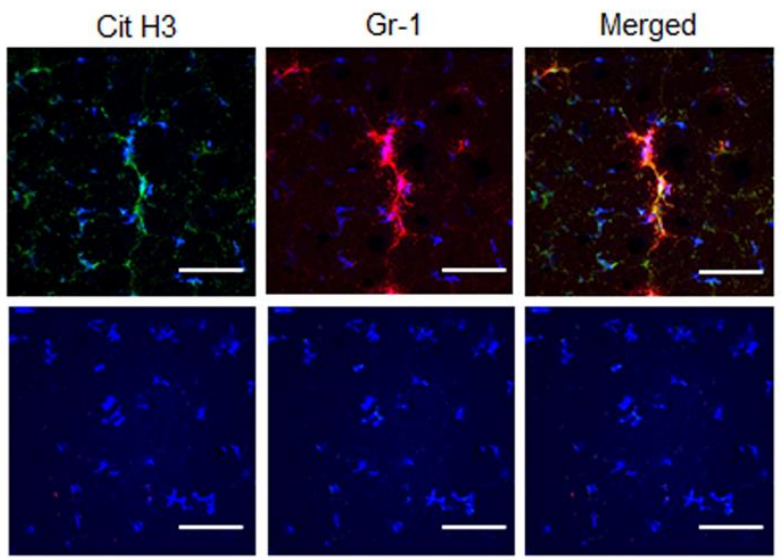

(d)

Anti Gr-1 lgG

$$
\frac{\text { Normal rat } \lg G}{\text { Cont Stimulated }} \frac{\text { Anti Gr-1 } \lg G}{\text { Cont Stimulated }}
$$
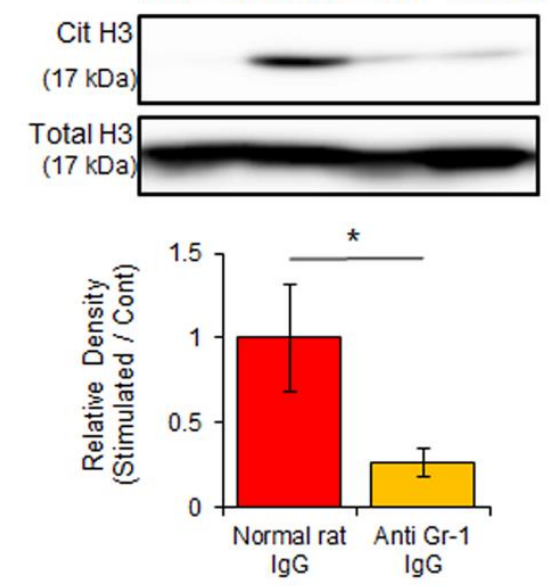

(e)

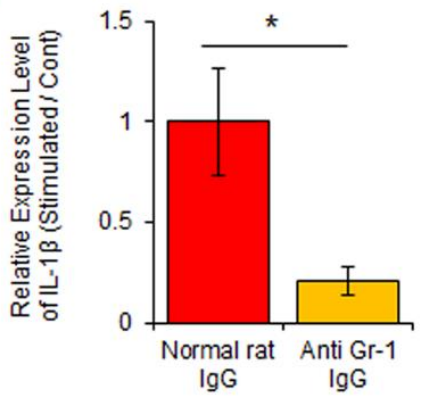


Figure 5

729

(a)

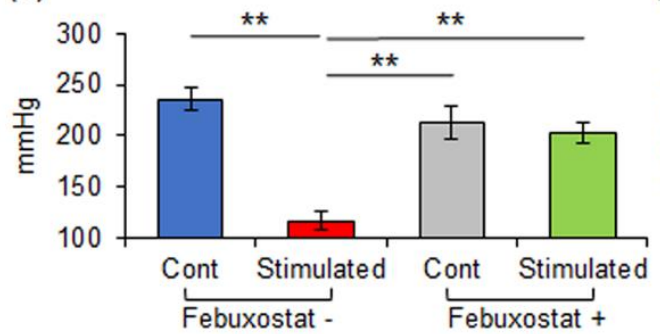

(b)

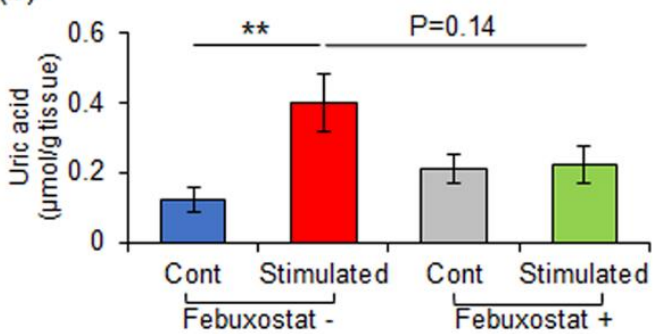

732

(c)

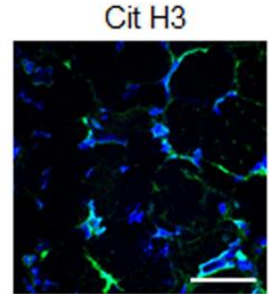

$\mathrm{Gr}-1$

Merged

733

Febuxostat-

734

735

Febuxostat+

736

(d)
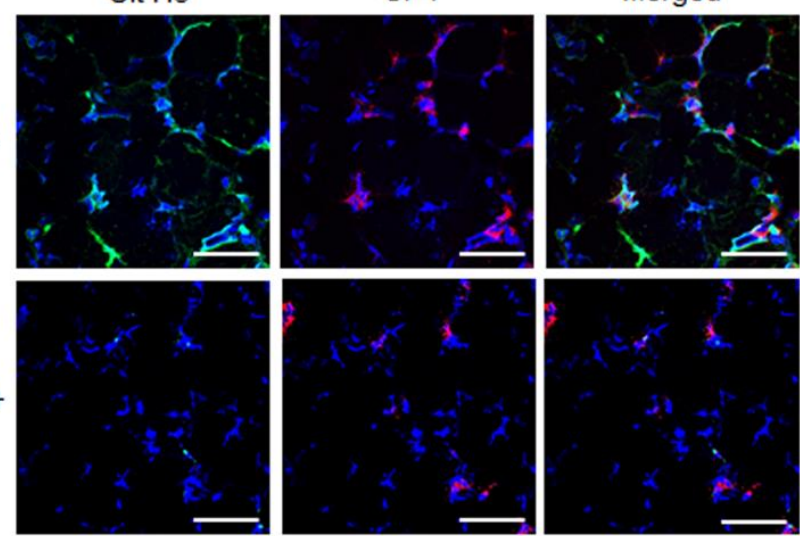

737

738

739

$\frac{\text { Febuxostat- }}{\text { Cont Stimulated }} \frac{\text { Febuxostat }+}{\text { Cont Stimulated }}$
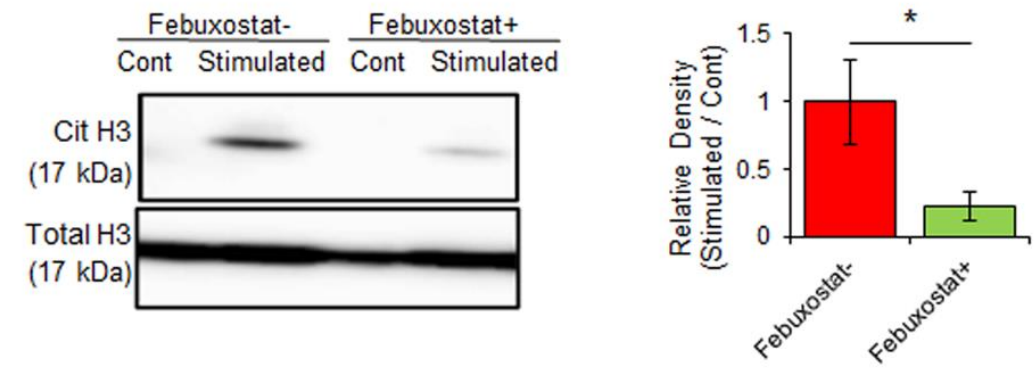

(e)

740

Febuxostat- Febuxostat+

(f)

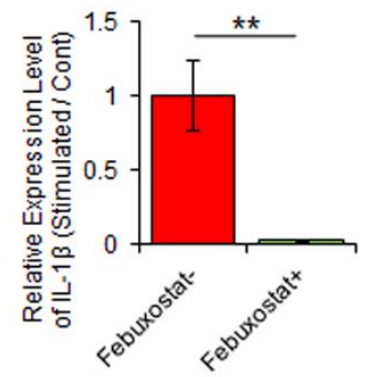

743 
$744 \quad$ Figure 6

745

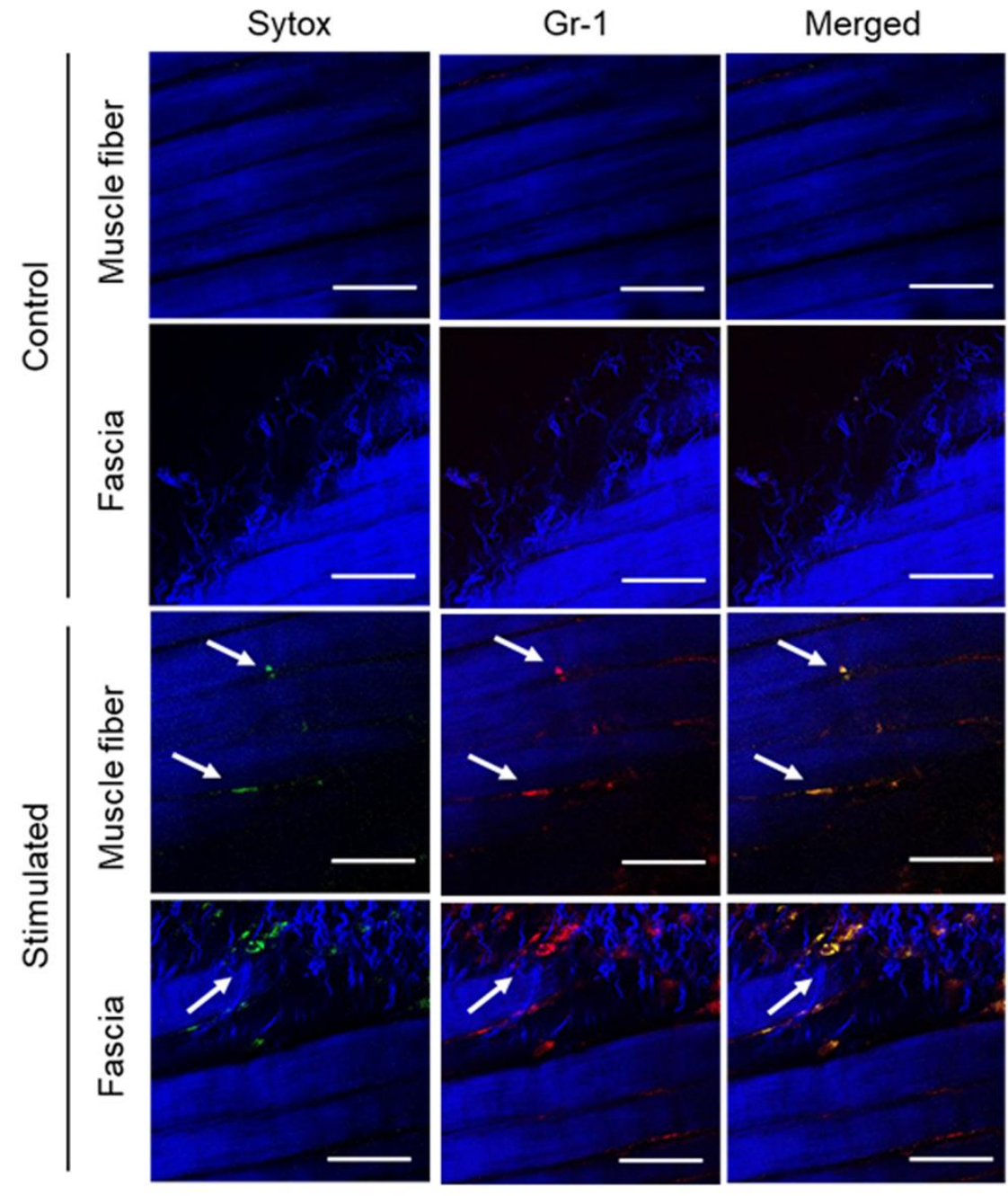

756

757

758

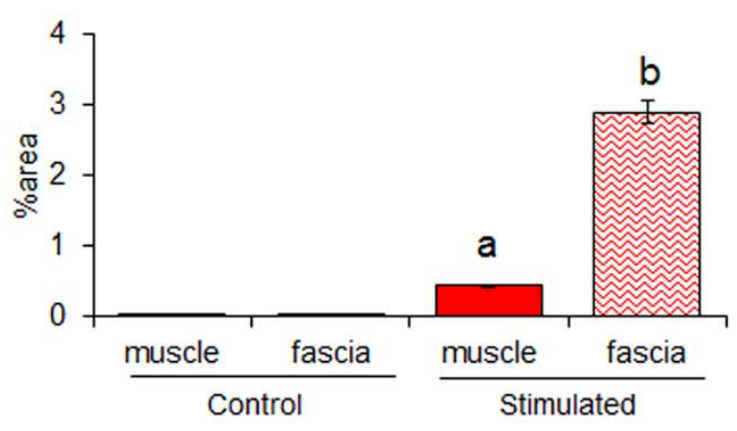

759 
Figure 7

761

Excessive muscle contraction by electrical stimulation

762

763

Muscle cell damage

764

765

766

767

768

769

Gr-1]

antibody

770
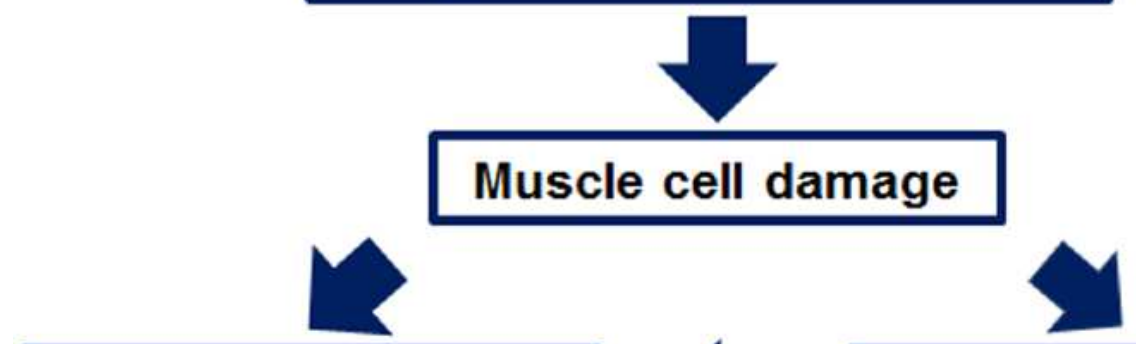

Febuxostat

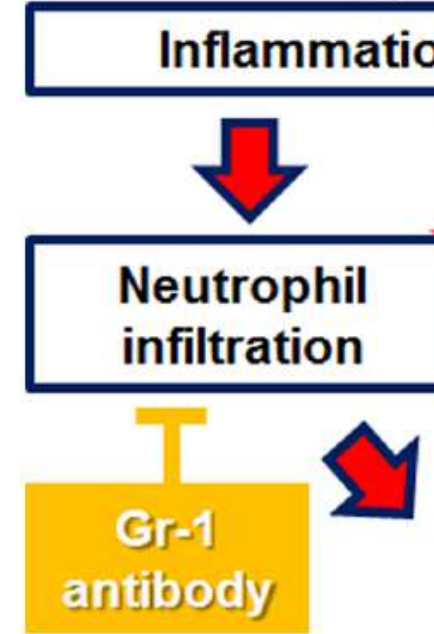

Uric acid production

Cytokine
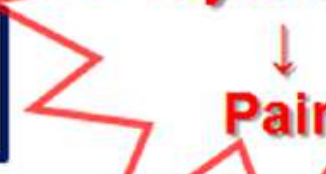

Pain

Nucleic acid metabolism

$+$
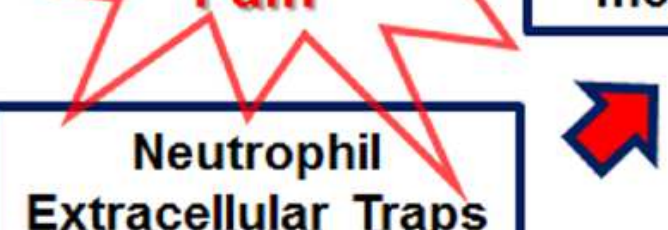

Extracellular Traps

\section{DNase}

771

772 


\section{Supplementary Files}

This is a list of supplementary files associated with this preprint. Click to download.

- Supplementalmovie1acont.mpg

- Supplementalmovie1bstimulated.mpg

- SupplementaryFigure.pdf 